\title{
Actualización:
}

\section{Tratamiento de la alopecia androgenética}

Treatment of androgenetic alopecia

Jorge Kontos*

\begin{abstract}
Resumen
La alopecia es un motivo de preocupación muy frecuente en hombres y mujeres. Desde hace muchos años se ha intentado encontrar, con escaso éxito, un tratamiento eficaz.

Este artículo describe las causas y las formas de detectarlas a través del interrogatorio y el examen físico, para poder emplear con seguridad los mejores tratamientos disponibles para la alopecia androgenética: minoxidil tópico y finasteride oral.
\end{abstract}

\section{Abstract}

Alopecia is a frequent health concern that affects both men and women. A succestul treatment has been searched for many years.

This article deals with causes that can be detected through questioning and physical examination in order to treat patients with androgenetic alopecia in the best possible way: topic minoxidil and oral finasteride.

Palabras clave: alopecía androgenética, tratamiento. Key words: androgenetic alopecia, treatment.

Kontos J. Tratamiento de la alopecía androgenética. Evid. actual. práct. ambul; 11(4): 120-122, Jul-Ago.2008.

\section{Introducción}

La caída del cabello (alopecia) afecta a hombres y mujeres de todas las edades e incide en forma negativa en la autoestima de los pacientes. Desde hace muchos años las personas afectadas por este problema utilizan muchos tratamientos y gastan mucho dinero para evitar o retardar la caída del cabello.

Hasta dos tercios de los hombres pueden tener alopecia androgénica, con repercusión psicológica en la mitad de ellos ${ }^{1}$. En este artículo revisaremos el ciclo de crecimiento normal del cabello y sus principales patologías, con el objetivo de que nos permita elegir el mejor tratamiento para nuestro paciente.

\section{Crecimiento normal del cabello}

El pelo del cuero cabelludo crece aproximadamente $0,35 \mathrm{~mm}$ cada día, desprendiéndose unos cien cabellos diariamente y aun más con el uso de shampoo².

Cada cabello atraviesa tres etapas de crecimiento. Entre 85 y 95\% del cabello se encuentra en la fase anágena, permaneciendo los folículos en esta etapa durante dos a seis años. La fase siguiente es la catágena, dura de dos a cuatro semanas e implica entre 2 y $35 \%$ de los folículos. Por último, se describe la fase telógena en la cual 10 a $15 \%$ de los folículos se encuentra en un periodo "de descanso" que dura tres meses. Al terminar esta fase, el cabello muerto es eliminado de la piel, dejando un nódulo sólido de color blanco en su zona de inserción. Luego el ciclo se repite. Ver figura 1.

Estas tres etapas se producen en forma simultánea y como mencionamos, a diferencia de los folículos de otros animales, los de los humanos no están al mismo tiempo en el mismo ciclo, por lo que este proceso puede no ser percibido.

Figura 1: ciclo del cabello humano.

ELIMINACION Del PELO MUERTO

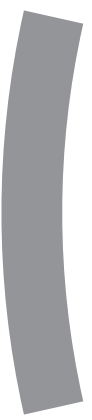

FASE TELOGENA (DESCANSO)

3 MESES 12 A $15 \%$
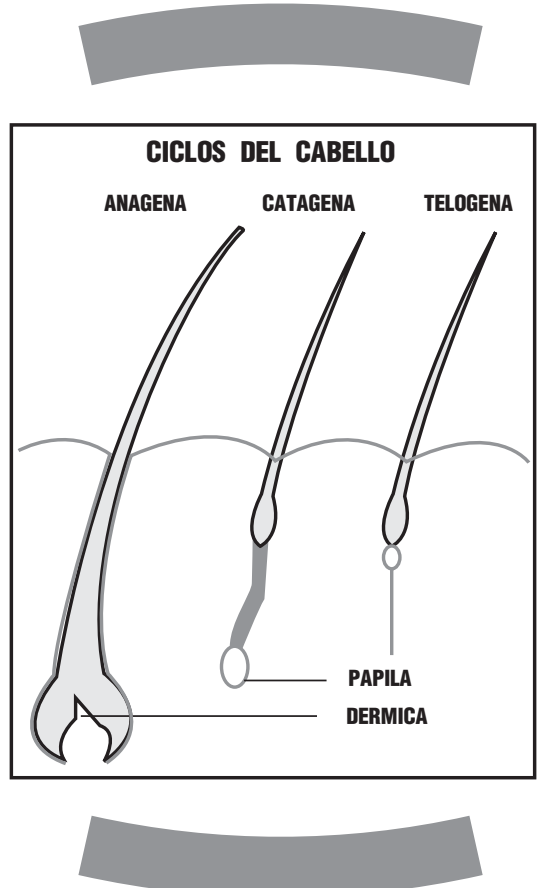

FASE ANAGENA

(CRECE)

2 A 6 AÑOS

$90 \%$

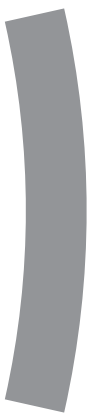

FASE CATAGENA
(TRANSICION)
2-4 SEMANAS

2 A $35 \%$

* Medico de Familia. Hospital Rawson. Córdoba Capital. jorgekontos@yahoo.com.ar 


\section{Fisiopatogenia}

Aunque la alopecía androgenética sea la causa más común de calvicie en hombres, las causas patológicas deben ser consideradas en el manejo de este problema de salud. Se las puede dividir en cicatrizales y no cicatrizales (ver tabla1) observándose las primeras frecuentemente en pacientes con problemas infecciosos o del tejido conectivo.

Tabla 1: causas de alopecia.

\begin{tabular}{l|l}
\multicolumn{1}{c|}{ No cicatrizales } & \multicolumn{1}{c}{ Cicatrizales } \\
\hline - Alopecia Androgenética & - Infecciones \\
- Efluvio felogeno & - Dermatopatías inflamatorias no infecciosas \\
- Tricotilománia & (ej. alteraciones del tejido conectivo como \\
- Alopecia por tracción & lupus eritematoso sistémico). \\
- Alopecia areata & - Infecciones \\
- Sifilis secundaria & - Agentes físicos y químicos \\
- Aíteraciones congénitas & - Neoplasias \\
& - Alteraciones congénitas \\
\hline
\end{tabular}

Resumido de: Fitzpatrick y col. Dermatologia en Medicina General. 6 Ed. Tomo 1. p729 Editorial Panamericana 2005

\section{Evaluación inicial y diagnóstico}

Un examen físico y un interrogatorio dirigido habitualmente muestran la causa de la caída de cabello, siendo importante detectar las causas de alopecia no androgenética.

Se debe preguntar cuándo comenzó la alopecia y si ésta fue gradual o abrupta, si el cabello se nota más delgado y por esto se ve más el cuero cabelludo, si la caída del pelo fue masiva en una zona o si fue de tipo difusa.

El importante interrogar sobre el consumo de medicación como anticoagulantes, anticonvulsivantes, antidepresivos o beta-bloqueantes; y sobre la presencia de enfermedades concomitantes como hiper o hipotiroidismo y/o deficiencia de hierro.

La pérdida de cabellos en forma de parches puede estar asociada con tinea capitis, lupus eritematoso y con alopecía areata. Una historia de pérdida abrupta de cabello luego de un episodio estresante de tipo físico, mental o emocional -dentro de los tres a seis meses de ocurrido- es indicativa de efluvio telógeno.

A medida que el patrón de calvicie se va desarrollando, los cabellos de las áreas afectadas se hacen más cortos y finos, despigmentándose con los sucesivos ciclos.

Una historia familiar de calvicie y la progresión de la pérdida de cabello en "M" sugieren alopecia androgenética. Este patrón de alopecia es hereditario (probablemente autonómico dominante) y dependiente de los andrógenos, relacionado con la dihidrotestosterona (DHT) de modo especifico ${ }^{3}$. La DHT es un metabolito de la testosterona por lo que los hombres con defectos genéticos de la 5 alfa-reductasa, la enzima que convierte la testosterona en DHT, no tienen calvicie con patrón masculino ${ }^{4}$.

En la alopecia androgenética como fue adelantado previamente los pelos se hacen más finos en un patrón de "M", ocurriendo esto en el vértex y en las entradas temporales que progresivamente terminan por despoblar al cuero cabelludo, a excepción de las zonas marginales que no son sensibles a la acción androgenética. Este patrón revela la distribución de los folículos andrógeno-sensitivos en la mayoría de las personas ${ }^{5}$.
Una técnica para evaluar cualquier tipo de caída es la tracción del cabello. Esta simple técnica consiste en asir manualmente un grupo de 50 a 100 pelos del cuero cabelludo y aplicar una tracción suave de un sólo movimiento desde la base hasta las puntas y repetirlo en varias zonas del cuero cabelludo ${ }^{6}$. Los cabellos deben ser traccionados firmemente pero con delicadeza. Un resultado negativo a dicha prueba -obtener seis pelos o menos- indica una caída normal de cabello. Por el contrario, se debe considerar que las áreas en las que ésta resulta positiva -más de seis cabellos por tracción- tienen un incremento en la caída del cabello. Los pacientes no deberían usar shampoo las durante las 24 horas previas a la realización de la prueba $^{7}$.

Antes de inciciarse el tratamiento, el diagnostico definitivo -principalmente en los casos de tipo cicatrizal- suele realizarse por medio de una biopsia. La alopecia androgenética es de tipo no cicatrizal.

\section{Tratamientos medicos}

\section{Minoxidil}

El minoxidil tópico es un tratamiento efectivo para hombres y mujeres con alopecia androgenética, siendo recomendado en primera línea por la Asociación Estadounidense de Dermatología ${ }^{8}$. Es de venta libre en solución tópica al $2 \%$-autorizado para su uso en mujeres y hombres- y al $5 \%$, que sólo debería ser usado por hombres. Si bien parece actuar a nivel de los folículos, su mecanismo de acción es desconocido. Hay datos limitados en mujeres que indican una mayor eficacia de la solución al 5\%, pero algunas de estas pacientes desarrollan mayor crecimiento del vello facial con la solución mas concentrada ${ }^{9}$.

Se deben aplicar 20 gotas de minoxidil dos veces por día sobre el cuero cabelludo, no siendo necesario masajear el cuero cabelludo. Las soluciones contienen productos que pueden dejar manchas en el cabello y que se van con el lavado.

Se recomienda evaluar su eficacia luego de un año de uso. En este sentido puede ser útil tomar fotografías al inicio del tratamiento y cada cuatro meses. No tiene eficacia si se lo utiliza en áreas sin cabellos y tiene pocos efectos adversos. El tratamiento con minoxidil al $5 \%$ se asoció con una respuesta terapéutica más temprana pero se asocia con mayor incidencia de prurito e irritación local que la solución al $2 \%$. Entre los más comunes se encuentra la dermatitis de contacto. No afecta la tensión arterial ni el pulso, aunque los pacientes con antecedentes de enfermedad cardiovascular deben ser advertidos si tienen taquicardia, edemas o ganancia de peso, ya que si la piel no está intacta puede haber absorción sistémica del producto.

El candidato ideal para utilizar minoxidil sería un paciente con un área sin pelos menor a $10 \mathrm{~cm}$ de diámetro, localizada en el vértex y de menos de cinco años de evolución. El minoxidil no es útil para tratar la alopecia frontal. Los hombres que suspendieron su uso tuvieron una rápida caída del cabello ganado durante el tratamiento, y a los tres meses hubo un recuento de pelos por debajo del que tenían antes de iniciar el tratamiento. En Argentina, el costo mensual del tratamiento mensual varía entre el equivalente a u\$s13 y u\$s18.

\section{Finasteride}

Otra opción en varones es usar finasteride. Esta droga inhibe la enzima 5-alfa-reductasa, que convierte la testosterona en dihidrotestosterona, agente que sería el metabolito activo sobre el cuero cabelludo. Originalmente se vendía sólo en comprimi- 
dos de $5 \mathrm{mg}$ para el tratamiento de la hipertrofia prostática benigna, ahora disponemos de presentaciones de $1 \mathrm{mg}$, para el tratamiento de la alopecia.

El finasteride demostró detener la caída del cabello en un 60 a $80 \%$ de pacientes y aumentar un 30 a $40 \%$ el crecimiento del pelo. El efecto de la droga se nota a los seis meses de comenzada, siendo mayor su efecto en el vértex que en el área frontal ${ }^{10}$. Su mayor efectividad se da en pacientes con inicio reciente de la caída del cabello. Luego de dos años de uso, siguieron perdiendo pelo el $72 \%$ de los que habían usado placebo y solo el $17 \%$ de los que habían usado finasteride. ${ }^{4}$

Tiene pocos efectos adversos, entre los que se pueden describir disminución de la libido, disfunción sexual eréctil y disminución del volumen eyaculatorio, sobre los cuales hay que interrogar al paciente durante los controles. La dosis es de $1 \mathrm{mg}$ una vez al día por un mes cuesta en Argentina un mínimo de u\$s13 mensuales.

Esta droga no ha sido aprobada por la Agencia de Drogas y Alimentos de EE.UU. (en inglés FDA) para usar en mujeres ya que no demostró eficacia ${ }^{11}$. Además, las mujeres que pudieran estar embarazadas no deberían consumir finasteride, ya que puede traer defectos en fetos de sexo masculino.

No disponemos de estudios que hayan comparado directamente minoxidil con finasteride en pacientes con alopecia, habiendo evaluado el uso combinado sólo un estudio de casos y controles. Nuestra observación clínica sugiere que la adherencia al tratamiento es mayor con finasteride ya que es más fácil de usar.

En las mujeres con signos de hiperandrogenismo se deben medir los niveles de dehidroepiandrostenediona, prolactina y testosterona. Como tratamientos adicionales para las mujeres, se puede utilizar anticonceptivos orales o espironolactona a dosis de 100 a $200 \mathrm{mg}$ por día.

La espironolactona es un antagonista de la aldosterona con efectos antiandrogénicos. Es utilizada para el tratamiento del hirsutismo y puede retrasar la pérdida de cabello en mujeres con alopecia androgenica, pero no estimula el crecimiento de cabello ${ }^{6}$.

La tabla 2 resume las principales características de los tratamientos más usados.

Tabla 2: comparación de los diferentes tratamientos para la alopecia androgenética.

\begin{tabular}{|c|c|c|c|c|}
\hline Droga & Dosis & $\begin{array}{c}\text { Costo anual en } \\
\text { Argentina }\end{array}$ & Ventajas & Desventajas \\
\hline Minoxidil al $2 \%$ & $1 \mathrm{~mL}$ dos veces por día & $\approx u \$ s 150$ & $\begin{array}{l}\text { Se puede usar en hombres y mujeres } \\
\text { con moderada efectividad }\end{array}$ & $\begin{array}{l}\text { No sirve para la alopecia frontal. Puede } \\
\text { tener efectos adversos locales. } \\
\text { El cabello núevo se pierde al discontinuar } \\
\text { el tratamiento }\end{array}$ \\
\hline Minoxidil al $\mathbf{5} \%$ & $\begin{array}{c}\text { (20 gotas o seis aplicaciones de } \\
\text { Spray) }\end{array}$ & $\approx u \$ s 150$ & Más efectivo que la solución al $2 \%$ & $\begin{array}{l}\text { No sirve para la alopecia frontal. Puede } \\
\text { tener efectos adversos locales. } \\
\text { El cabello nuevo se pierde al discontinuar } \\
\text { el tratamiento }\end{array}$ \\
\hline Finasteride & $1 \mathrm{mg}$ una vez al día & $\approx u \$ s 220$ & $\begin{array}{l}\text { Los efectos adversos son infrecuentes. } \\
\text { Es efectivo en vertex y algo efectivo } \\
\text { para zona frontal. } \\
\text { Se utiliza por vía oral }\end{array}$ & $\begin{array}{l}\text { Sólo se puede usar en hombres. } \\
\text { El cabello nuevo se pierde al discontinuar } \\
\text { el tratamiento. Puede afectar los valores de } \\
\text { antgeno prostático específico. }\end{array}$ \\
\hline
\end{tabular}

Modificada de: Fitzpatrick y col. Dermatologia en Medicina General. 6ª Ed. Tomo 1. p729 Editorial Panamericana 2005.

Referencias

1. Nielson Ty col. Alopecia: diagnosis and management. Am Fam Physician 1995;51:1513-22,1527-8.

2. Sperling L y col. Hair diseases. Med Clin North Am 1998;82:1155-69.

3. Fitzpatrick y col. Dermatologia en Medicina General. $6^{\mathrm{a}}$ Ed. Tomo 1 p729 Editorial Panamericana 2005

4. Scow D y col. Medical treatments for balding in men. Am Fam Physician 1999 Apr 15;59(8):2189-94, 2196

5. Springer K y col. Common hair loss disorders. Am Fam Physician 2003: 68:1: 93-1002

6. Olsen E: Clinical tools for assesing hair loss: Diagnosis and treatmente, edited by EA Olsen. New York, Mc Graw Hill, 2003

7. Thiedke C. Alopecia in women. Am Fam Physician 2003: 67:5: 1007-1014

8. Drake L y col. Guidelines of care for androgenetic alopecia. American Academy of Dermatology. J Am Acad Dermatol 1996;35(3 pt 1):465-9.

9. Lucky A y col. A randomized, placebo-controlled trial of 5\% and 2\% topical minoxidil solutions in the treatment of female pattem hair loss. J Am Acad Dematol 2004; 50:541.

10. Rubinstein E y col. Alopecia. Capítulo 249. En: Adolfo Rubinstein y Sergio Terrasa. Medicina Familiar y Práctica Ambulatoria. 2da ed. Ed.Médica Panamericana. Buenos Aires 2006.

11. Price $V y$ col. Lack of efficacy of finasteride in postmenopausal women with androgenetic alopecia. J Am Acad Dermatol 2000;43 (5pt1):768-76. 